\title{
Self-Healing Performance of Rubber-Modified Asphalt
}

\author{
Yang Luo*, Guocui Teng \\ School of Construction Engineering, Jilin University, Changchun 130026, China
}

Corresponding Author Email: luoyang19@mails.jlu.edu.cn

https://doi.org/10.18280/eesrj.080202

Received: 10 March 2021

Accepted: 25 May 2021

\section{Keywords:}

road engineering, self-healing, modified asphalt, molecule simulation, rubber asphalt, molecular dynamics

\begin{abstract}
To figure out the effect of rubber modifiers on the self-healing performance of asphalt this study took Eucommia rubber (TPI, trans-polyisoprene) and natural rubber (NR) as asphalt modifiers and employed the molecule simulation software to analyze the selfhealing performance of asphalt in the microscale. At first, a model of basic asphalt system was established in the molecule simulation software, and three parameters of density, solubility and mean square displacement (MSD) were used to verify that the established model can act as the representative of asphalt molecules; then, several self-healing models of basic asphalt and rubber-modified asphalt were constructed to compare the properties of TPI and NR. In the experiment, the temperature was set to $303.15 \mathrm{~K}, 313.15 \mathrm{~K}$, $323.15 \mathrm{~K}, 333.15 \mathrm{~K}$, and $343.15 \mathrm{~K}$, respectively, and a $10 \AA(1 \AA=0.1 \mathrm{~nm})$ vacuum layer was taken as the microcrack of the asphalt, then the entire self-healing process of the models was analyzed, and the diffusion coefficient and energy change of the models were calculated. The experimental results revealed that, the NR-modified asphalt outperformed TPI in self-healing performance; for the NR-modified asphalt, as the temperature rises, the intermolecular non-bond energy changes, the molecule diffusion ability enhances, which can accelerate the self-healing progress of the microcracks in the asphalt.
\end{abstract}

\section{INTRODUCTION}

With the progress of industrialization in China, the demand for rubber is increasing significantly, and NR is in short supply, in this context, TPI is often used as a substitute of NR. TPI is a type of natural high molecular polymer, and its main chemical composition is trans-1,4-polyisoprene, which is the same as NR (cis-1,4-polyisoprene), and the two are isomers [1]. Scholar Xu et al. [2] used molecular dynamics to compare pure TPI and TPI/NR blends and proved that the blends have better static mechanical properties and dynamic tensile fatigue resistance. Zhang et al. [3] used molecular dynamics to analyze the glass transition temperature of TPI and NR and verified the results through DSC experiment. Wang [4] believes that elastic recovery and the existence of hydrogen bond networks are the main mechanisms for the self-repair of rubber system. Xuan et al. [5] analyzed the self-healing performance of rubber asphalt based on viscoelastic theory, and adopted temperature difference displayer and time scanner to compare the size of the stress and the impact of damage degree on the self-healing performance. This study employed the Materials studio software to study and compare the selfhealing performance of NR-modified asphalt, TPI-modified asphalt, and basic asphalt in the microscale.

\section{CONSTRUCTION AND VERIFICATION OF ASPHALT MODEL}

\subsection{Modeling}

Asphalt has a very complex molecular structure, which is often divided into three components, four components, or other types by field scholars, and each component can be subdivided into multiple molecules to represent its properties; the molecules include various chemical structures such as benzene rings, saturated hydrocarbons, and saturated hydrocarbon branches. Rogel and Carbognani [6] believes that the benzene ring group and short branches constitute the asphaltene molecules, and he used 8 asphaltene models to perform molecular dynamics analysis. Su et al. [7] divided asphalt into three components to simulate the asphaltene molecules, and tried to use the $\mathrm{C}_{22} \mathrm{H}_{46}$ molecule to represent the oil component. Zhang and Greenfield [8, 9] analyzed the structure of asphaltene molecules and successively proposed two molecular configurations, they introduced a polar atom, the sulfur atom, and proposed a new four-component asphalt model based on the AAA-1 asphalt components. $\mathrm{Li}$ and Greenfield $[10,11]$ used four-component structure to construct asphalt molecules and expanded the component molecules into 12 molecules (see Table 1); each component was replaced by a variety of chemical molecules, which can better describe the properties of each component, and the constructed asphalt molecular model is representative. This study adopted the 4component and 12-molecule structure (see Figure 1) to build the basic asphalt model (Figure 2). The asphalt model was constructed in Materials studio, and the validity of the model was verified by calculating the model parameters such as solubility, density, and MSD.

\subsubsection{Modeling process}

For a single molecule, after undergoing 5 temperature cycles between $300 \mathrm{~K}-500 \mathrm{~K}$, it's subject to geometric optimization and annealing treatment; then for the optimized molecule, under the NVT ensemble (N-molecular number, V- 
molecular volume, T-temperature, constant), the Compass-II force field and atom-based van der Waals (VDW) analysis, the steady state molecule was obtained (see Figure 3). For the 4 components and 12 molecules, the Construction Task in the Amorphous Cell module was used to construct the periodic model of asphalt molecules. The model was subject to geometric optimization and dynamics optimization, and then subject to NVT ensemble optimization and NPT ensemble optimization, after running for a time period of $200 \mathrm{ps}$, a stable system was formed.

Table 1. A summary of the 4 components and 12 molecules

\begin{tabular}{ccccc}
\hline $\begin{array}{c}\text { Component } \\
\text { type }\end{array}$ & No. & $\begin{array}{c}\text { Molecular } \\
\text { formula }\end{array}$ & $\begin{array}{c}\text { Molecular } \\
\text { mass }\end{array}$ & $\begin{array}{c}\text { Number of } \\
\text { molecules }\end{array}$ \\
\hline \multirow{5}{*}{ Colloids } & $\mathrm{I}$ & $\mathrm{C}_{40} \mathrm{H}_{50} \mathrm{~N}$ & 553.9 & 4 \\
& $\mathrm{Ii}$ & $\mathrm{C}_{36} \mathrm{H}_{57} \mathrm{~N}$ & 503.8 & 4 \\
& $\mathrm{Iii}$ & $\mathrm{C}_{18} \mathrm{H}_{10} \mathrm{~S}_{2}$ & 290.3 & 15 \\
& $\mathrm{Iv}$ & $\mathrm{C}_{40} \mathrm{H}_{60} \mathrm{~S}_{2}$ & 468.8 & 4 \\
Saturates & V & $\mathrm{C}_{29} \mathrm{H}_{50} \mathrm{O}$ & 350.6 & 5 \\
& Vi & $\mathrm{C}_{35} \mathrm{H}_{62}$ & 483.8 & 4 \\
Aromatics & Vii & $\mathrm{C}_{30} \mathrm{H}_{62}$ & 422.8 & 4 \\
& $\mathrm{Viii}$ & $\mathrm{C}_{35} \mathrm{H}_{44}$ & 464.7 & 11 \\
& $\mathrm{Ix}$ & $\mathrm{C}_{30} \mathrm{H}_{46}$ & 406.6 & 13 \\
Asphaltene & $\mathrm{X}$ & $\mathrm{C}_{42} \mathrm{H}_{54} \mathrm{O}$ & 595.0 & 3 \\
& $\mathrm{Xi}$ & $\mathrm{C}_{51} \mathrm{H}_{62} \mathrm{~S}$ & 707.1 & 3 \\
& $\mathrm{Xii}$ & $\mathrm{C}_{66} \mathrm{H}_{81} \mathrm{~N}$ & 886.3 & 2 \\
\hline
\end{tabular}

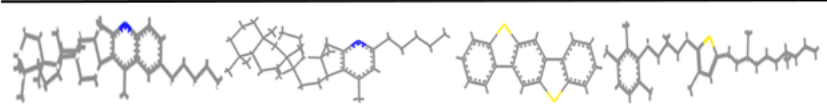

(i)

(ii)

(iii)

(iv)

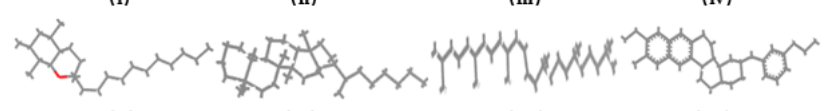

(v)

(vi)

(vii)

(viii)

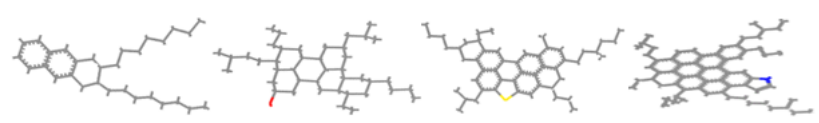

(ix)

(x)

(xi)

(xii)

Figure 1. A diagram of the 4-component 12-molecule structure

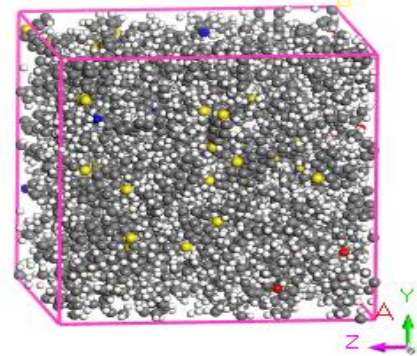

Figure 2. Model of basic asphalt
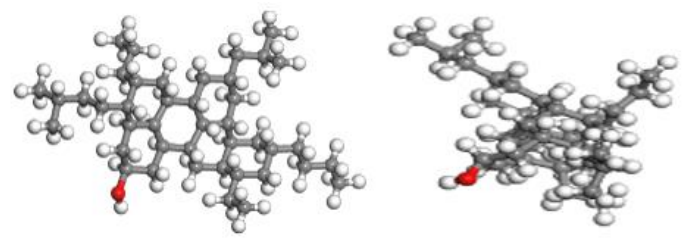

Figure 3. Comparison of molecule before and after optimization: (a) Colloidal molecule before optimization; (b) Colloidal molecule after optimization

\subsection{Verification of the asphalt model}

\subsubsection{Radial distribution function (RDF)}

RDF can describe the orderly distribution of asphalt molecules in space. Within a certain distance, the distribution is orderly, after exceeding the distance, the distribution becomes disorderly.

$$
g(r)=\frac{1}{4 \rho \pi r^{2}} \frac{\sum_{i=1}^{r} \sum_{j=1}^{n} \Delta N(r \rightarrow r+\xi r)}{N \times T}
$$

where [12], $\rho$ represents the system density $\left(\mathrm{Kg} / \mathrm{cm}^{3}\right) ; \mathrm{r}$ represents the distance between ions $(\mathrm{m})$; $\mathrm{N}$ represents the total number of particles; $\Delta \mathrm{N}$ represents the number of molecules in the system interval; $T$ represents the system calculation time (ps); $\xi$ is an infinitesimal constant.

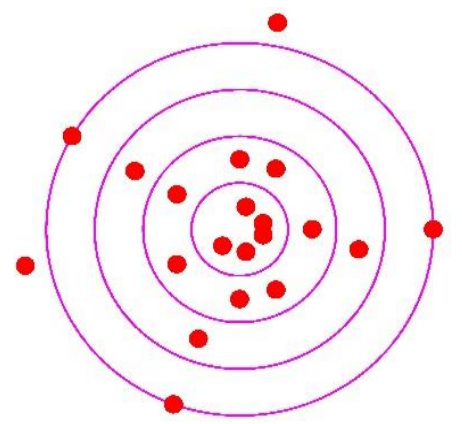

Figure 4. A diagram of the concept of the RDF of particles

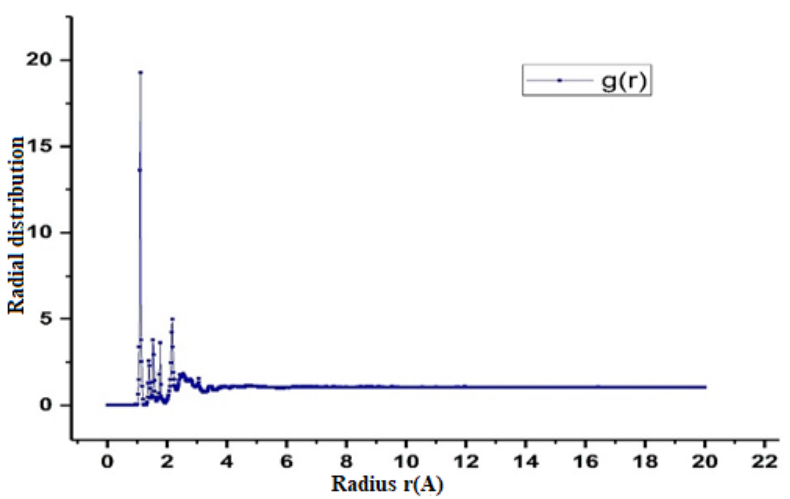

Figure 5. Radial distribution of basic asphalt particles

Figure 4 above shows that an asphalt molecule in the system is located at the center of a space sphere with a radius of $r$, and the atoms beyond the range of $r$ exhibit a disordered state. Figure 5 gives a diagram of the RDF of basic asphalt system, in the figure, atoms gather at the position of $\mathrm{r}=1 \AA(1 \AA=0.1 \mathrm{~nm})$; within the range with a radius of $1-5 \AA$, the atoms in the asphalt model distribute orderly; when the distance exceeds $5 \AA$, g(r) becomes very smooth and gradually approaches 1 , indicating that the spatial arrangement of the asphalt model shows disordered distribution at the far end, which is consistent with the actual spatial distribution of asphalt particles.

\subsubsection{Solubility}

The solubility is the degree of compatibility of multiple substances with each other after they are mixed. Wang et al. [13] used the solubility parameter to evaluate the compatibility of SBS modifier and asphalt material. Solubility is the degree 
of mixing of molecules in the mixture and the interaction of the functional groups, therefore it can be determined by the average energy. Cohesive energy density (CED) is the energy required for $1 \mathrm{~mol}$ of polymers per unit volume to overcome intermolecular forces and become gas, it can be calculated using the molecular simulation software and then the solubility parameter $\delta$ could be determined (such as Table 2):

$$
\begin{gathered}
C E D=E_{\text {coh }} / V \\
\delta=\sqrt{C E D}
\end{gathered}
$$

where, $E_{c o h}$ is the energy required for molecules in the system to diffuse to infinity; CED represents the cohesive energy density $\left(\mathrm{J} / \mathrm{cm}^{3}\right)$.

The density of the asphalt molecule model was calculated to be $0.99 \mathrm{~g} / \mathrm{cm}^{3}$, its deviation from the actual density value was within 5\%, which met the requirement and the solubility was within the experimental data range of asphalt, and this proved that the proposed asphalt molecule model can represent real asphalt molecules.

Table 2. Asphalt model parameter verification

\begin{tabular}{c|c|c}
\hline $\begin{array}{c}\text { Structural } \\
\text { parameter }\end{array}$ & Density $\left(\mathbf{g} / \mathbf{c m}^{\mathbf{3}}\right)$ & Solubility $\left(\mathbf{J} / \mathbf{c m}^{\mathbf{3}}\right)^{\mathbf{1 / 2}}$ \\
\hline $\begin{array}{c}\text { Simulation } \\
\text { result }\end{array}$ & 0.99 & 18.26 \\
\hline $\begin{array}{c}\text { Experiment } \\
\text { result [14, 15] }\end{array}$ & $1.02-1.04$ & $13.30-22.50$ \\
\hline
\end{tabular}

\section{INTRODUCTION OF MODIFIERS}

Rubber modifiers are widely used in road asphalt materials. They can improve the mechanical properties of asphalt and increase its viscosity; the rubber molecules are high molecular polymers formed by the polymerization of butadiene. The single chains of rubber molecules are optimized according to the steps of structural optimization of basic asphalt to obtain the stable structure. NR molecule has a cis structure, and the TPI molecule is an isomer of NR [16]. The structure of TPI molecule is trans-1,4-polyisoprene, and its linear structure is shown in Figure 6.<smiles>CC=C(C)CCC</smiles>

Figure 6. Structures of TPI and NR

\section{SELF-HEALING MODEL}

\subsection{Modeling}

The basic asphalt, the TPI-modified asphalt, and the NRmodified asphalt were modeled respectively. First the models were geometrically optimized at $300 \mathrm{~K}-500 \mathrm{~K}$ in the minimum energy state, and then under NVT ensemble and NPT ensemble, they were subject to model molecular optimization for $150 \mathrm{ps}$ so that the model systems were optimized to the greatest extent. For the stable systems of basic asphalt and modified asphalt, the Build Layers task in the Build module was used to build self-healing models, and a $10 \AA$ vacuum layer was used to represent the width of the self-healing microcrack. The 150ps NPT ensemble in MD of the Forcite module was run to simulate the self-healing phenomenon of asphalt, the COMPASSII force field was adopted, and the temperature was set to $303.15 \mathrm{~K}, 313.15 \mathrm{~K}, 323.15 \mathrm{~K}, 333.15 \mathrm{~K}$, and $343.15 \mathrm{~K}$ $\left(303.15 \mathrm{~K}=30^{\circ} \mathrm{C}\right)$ respectively, the pressure was $0.0001 \mathrm{Gpa}$ standard atmospheric pressure, the algorithm was optimized for 1,500,000 steps, the time step was $0.1 \mathrm{fs}$, and each structure was optimized for 5,000 steps. For the initial model systems, there're $\mathrm{OA}=37.58 \AA, \mathrm{OB}=37.58 \AA, \mathrm{OC}=117.55 \AA$ (In Figure 7 $\mathrm{C}$ : $\mathrm{OA}$ is the width of the model system, $\mathrm{OB}$ is the height, and $\mathrm{OC}$ is the length). In terms of the density data of the selfhealing process, the initial density of the structure system was $0.6170 \mathrm{~g} / \mathrm{cm}^{3}$, the system had a $10 \AA$ vacuum layer, in molecular dynamics, the volume of the system became smaller, the length of each edge (OA, OB, OC) was shortened, the molecules moved to the vacuum layer, and finally reached an equilibrium state, the density was $0.99 \mathrm{~g} / \mathrm{cm}^{3}$, which was consistent with the actual healing situation.

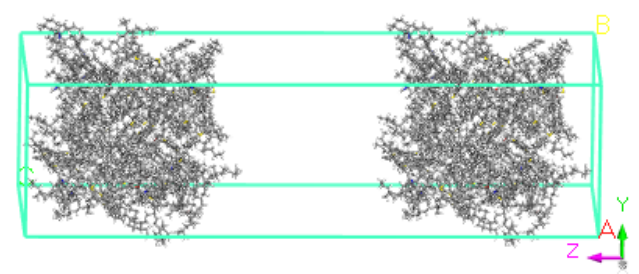

(c) Initial model

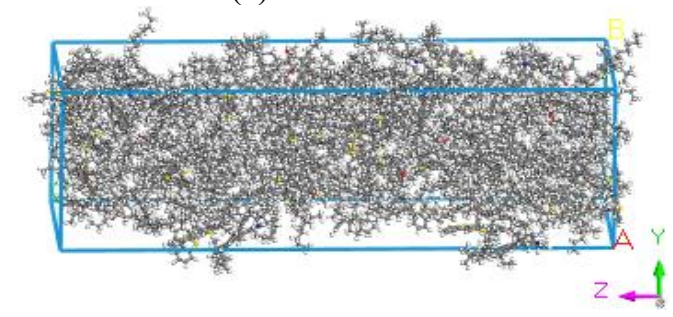

(d) Healed model

Figure 7. Models of basic asphalt (10尺) before and after healing

\subsection{Process of the healing model}

(1) Between 0-50ps, the asphalt self-healing system is in a turbulent stage at the beginning, the density curve decreases rapidly and then increases fast. In Figure 8, during the dynamic molecule movement, the asphalt molecules adjust their own temperature and convert the energy they have into kinetic energy to make the molecules be able to move; under the NPT ensemble, the density curve increases fast after the fluctuation period, and the molecules can move stably and rapidly, the density curve shows that the change of density is approximately proportional to the movement time.

(2) Between 50-100ps, the density curve changes slowly. In the self-healing model, molecules diffuse to the two ends of the vacuum layer. In the process of dynamic optimization of the system, the volume of the model is gradually compressed, and the density shows an upward trend. Asphalt molecules move stably, and the healing model performs structure restoration.

(3) Between 100-150ps, the healing density curve grows slowly, the molecules are well distributed in the system. In the 
healing model, the vacuum layer and the porous sections at both ends are distributed by the diffused molecules. In Figure 9 , the density curve fluctuates slightly and stabilizes at a certain value, the intermolecular structure and performance of the asphalt system return to the initial state.

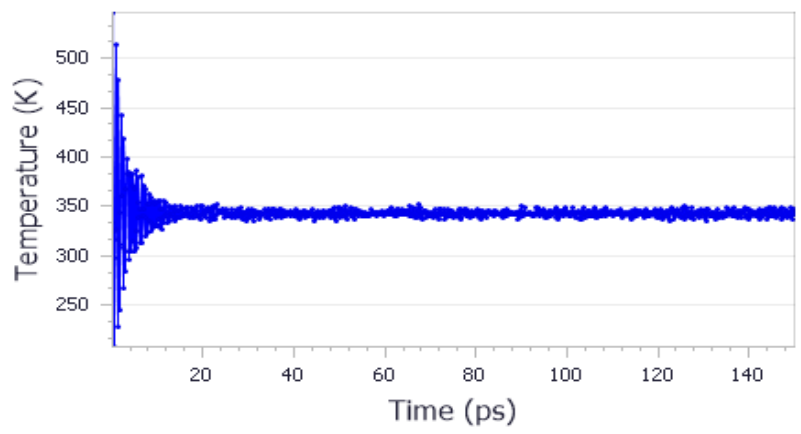

Figure 8. Temperature distribution of the basic asphalt $-10 \AA$ model

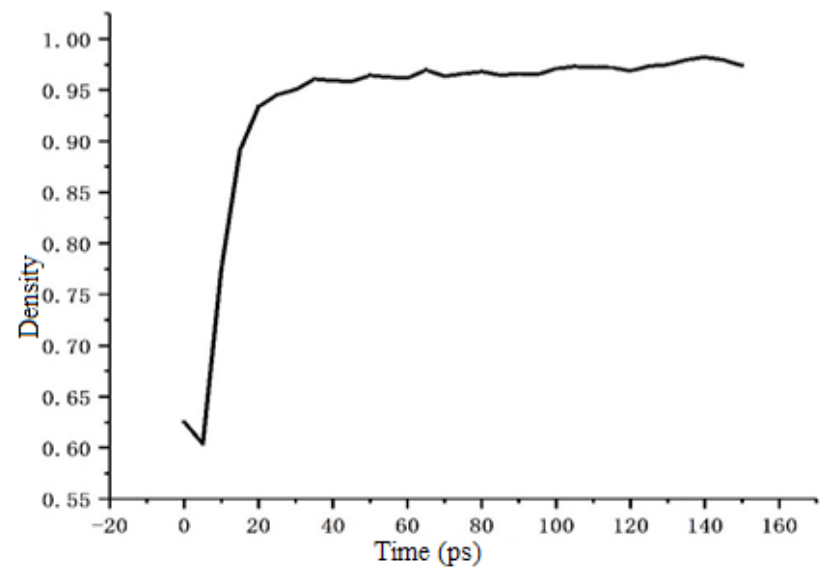

Figure 9. Density curve of $10 \AA$ asphalt system

\subsection{Energy change of the model system}

Figure 10 shows the energy change of the basic asphalt $10 \AA$ system running for $150 \mathrm{ps}$ in the NPT ensemble, as can be seen in the figure, between $0-20 \mathrm{ps}$, the potential energy and kinetic energy of the model are in a dynamic fluctuated state, the non-bond energy of the structure reduces to a certain value, the energy of asphalt molecules is given to the structure via temperature, in the healing system, the molecules move and distribute in the vacuum layer to restore the density and the structure; between 20-150ps, the structural energy is a balanced fluctuated value, indicating that the molecules in the system are in dynamic motion, and they restore the performance of the system.

According to Figure 11, in the basic asphalt $-10 \AA$ system, the spatial angle is set to 90 degrees, the structure is a rectangular shaped structure and remains unchanged during the model running period, the $\mathrm{OA}, \mathrm{OB}$, and $\mathrm{OC}$ of the system change, and the changes in the two directions of $\mathrm{OA}$ and $\mathrm{OB}$ coincide, which is the same with the change trend in the $\mathrm{OC}$ direction. Between 0-20ps, the changes of the three directions stretch to the greatest extent first and reduce to the dynamic equilibrium value latter, which are consistent with the change trend of density in Figure 10 (fast decreasing first and fast increasing latter), and this indicates that the structure fluctuates in the system due to the existence of energy molecules, which gives the asphalt molecules energy to diffuse in and heal the system; between 20-150ps, the asphalt healing model is in a dynamic distribution state, the system heals stably and restores to the initial state performance.

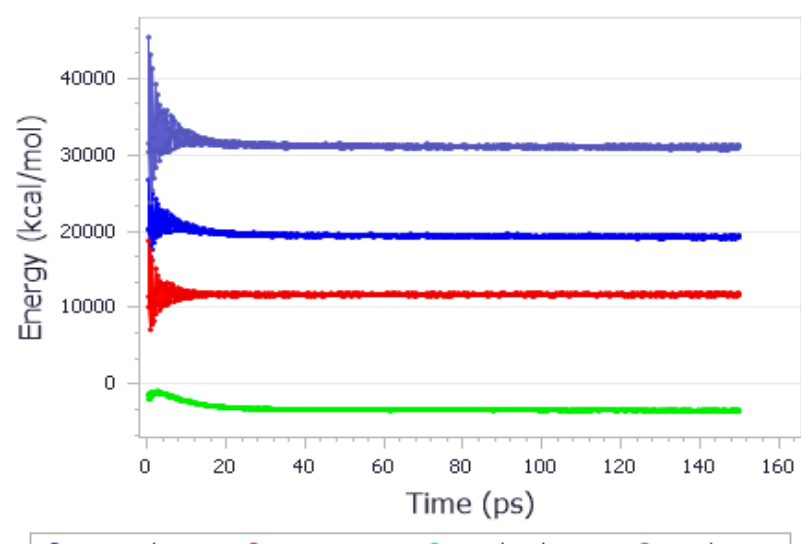

- Potential energy - - Kinetic energy - - Non-bond energy - - Total energy

Figure 10. Energy of basic asphalt $-10 \AA$ system
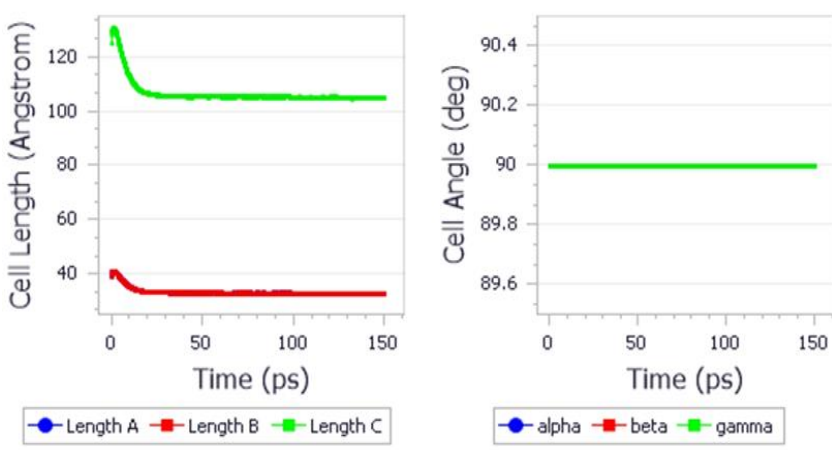

Figure 11. Cell length and angle of basic asphalt $-10 \AA$ system

\subsection{Diffusion coefficient}

According to the theory proposed by Einstein that the average of the sum of the squares of the random moving distances of particles is proportional to time, the derived diffusion coefficient formula is:

$$
<r^{2}>=6 D t+C
$$

where, $\left\langle\mathrm{r}^{2}\right\rangle$ is the average displacement; D is the diffusion coefficient; $\mathrm{C}$ is a constant; MSD is the mean square displacement of the model system, it represents the square of the distance between the position of a random particle in the asphalt molecule at time $t$ and the initial position of the particle.

$$
M S D_{(\Delta t)}=\left\langle[r(t-\Delta t)-r(t)]^{2}>\right.
$$

Then the formula of the diffusion coefficient is:

$$
D=\frac{1}{6 t} \lim _{t \rightarrow \infty} \frac{d M S D}{d \Delta t}
$$

In the dynamic simulation of self-healing models, the MSD analysis task in the Analysis of the Forceite module was adopted for each model and it was set to calculate the structure 
models every 5ps, the self-healing models of 1500000 simulation steps were calculated, and the MSD curves of each model are shown in Figure 11, according to the curves, we can know the entire MSD curves of the $10 \AA$ self-healing models of the NR and TPI systems under different temperatures of $303.15 \mathrm{~K}, 313.15 \mathrm{~K}, 323.15 \mathrm{~K}, 333.15 \mathrm{~K}$, and $343.15 \mathrm{~K}$.

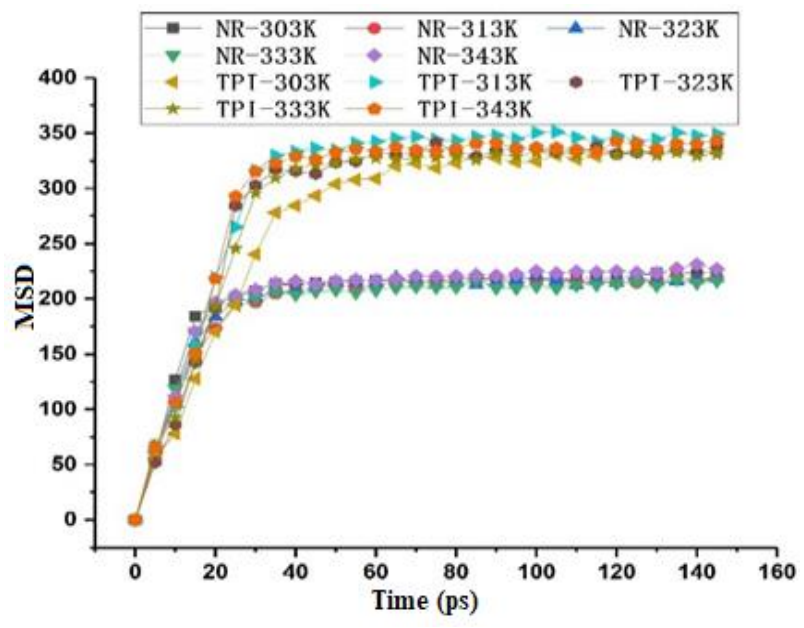

Figure 12. MSD curves of $10 \AA$ self-healing models under different temperatures

As shown in the Figure 12 above, MSD curves have three different slopes, respectively from 0 to $40 \mathrm{ps}$, from 40 to $80 \mathrm{ps}$, and from 80 to $150 \mathrm{ps}$. According to the curves of asphalt healing density, before 40ps is the asphalt self-healing process, at this stage, the diffusion of the asphalt self-healing model is mainly the recovery of strength and structure. After $40 \mathrm{ps}$, the structure restoration is gradually completed, at this stage, the diffusion is mainly the restoration of the properties of the asphalt, its properties restore and close to the original state. After $80 \mathrm{ps}$ is the asphalt self-diffusion stage after the healing is completed. The $0-40$ ps stage is the most representative stage and is selected for the calculation of diffusion coefficient, this stage is the longest one and a part of the asphalt healing process, and it is conductive to understanding the change of the diffusion coefficient of asphalt molecules during the asphalt healing process. Under the five temperature conditions, the healing curves of TPI models were above those of NR models, and the MSD values of TPI were greater than those of the NR models, indicating that TPI' diffusion performance is better than NR, but for the NR-modified asphalt, when MSD was $20 \mathrm{ps}$, the curves reached and fluctuated up and down around a stable value, and it reached the equilibrium value before TPI, adding rubber modifiers in the healing models has little impact on temperature. The diffusion coefficients of each model calculated by formulas (4), (5), and (6) are shown in Table 3 (unit: $\left.\left(\mathrm{m}^{-2} \cdot \mathrm{s}^{-1}\right)^{-1}\right)$ :

Table 3. Diffusion coefficients of each self-healing model $\left(\times 10^{-8}\right)$

\begin{tabular}{c|c|c|c|c|c}
\hline $\begin{array}{c}\text { Temperature } \\
\text { (K) }\end{array}$ & 303.15 & 313.15 & 323.15 & 333.15 & 343.15 \\
\hline TPI & 1.30 & 1.32 & 1.46 & 1.60 & 1.64 \\
\hline NR & 1.41 & 1.51 & 1.55 & 1.66 & 1.67 \\
\hline
\end{tabular}

Table 3 lists the diffusion coefficient values of rubbermodified asphalt under five temperature conditions. The values of the diffusion coefficient of the two types of modified asphalt increased with the temperature increment, indicating that rubber-modified asphalt has temperature sensitivity; under a same temperature, the diffusion coefficient of NRmodified asphalt is greater than that of TPI-modified asphalt, and its movement performance is better. The MSD curves of TPI-modified asphalt are above those of NR-modified asphalt, and the values are about 1.5 times. The viscosity of TPImodified asphalt is greater than that of NR-modified asphalt, the movement of the modified asphalt molecules promoted the healing of the microcracks in the models.

\section{CONCLUSIONS}

(1) Through three parameters of density, solubility, and MSD, the representativeness and validity of the basic asphalt model had been verified.

(2) A few self-healing models were constructed to simulate the microcracks and the healing process of the asphalt was simulated in the NPT ensemble, the healing process of TPImodified asphalt and NR-modified asphalt was analyzed; the balance points of MSD curves of NR-modified asphalt appeared before those of TPI-modified asphalt, indicating that the movement of NR-modified asphalt is better, and it can enhance the self-healing performance of the asphalt.

(3) As the temperature rises, the self-healing performance of rubber-modified asphalt is improved; under a same temperature, the self-healing performance improvement effect of NR is better than that of TPI

\section{REFERENCES}

[1] Zhu, F., Yue, H., Zu, E.F. (2005). Research and application of new functional material gutta percha. Journal of Anhui University Natural Science Edition, 29(3): 89-94.

[2] Xu, C., Yin, M.Z., Gao, W.J. (2012). Molecular dynamics simulation on mechanical properties of TPI/NR blends. China Adhesives, 21(7): 1-3.

[3] Zhang, H.J., Yue, H., Zhuang, C.Q., Liu, Q. (2011). Molecular Dynamics Simulation on the Glass Transition Temperatures of Tpi/Nr/Ldpe. Plastics, 40(1): 100-102, 127.

[4] Wang, W.Y. (2018). Design and synthesis of self-healing elastomer materials based on eucommic ulmoides gum and natural rubber. Shenyang University of Chemical Technology.

[5] Xuan, W.A., Xiong, J.P., Wei, W.F. (2020). Self-healing properties study of rubber asphalt based on viscoelastic theory. Highway, 65(1): 195-200.

[6] Rogel, E., Carbognani, L. (2003). Density estimation of asphaltenes using molecular dynamics simulations. Energy \& Fuels, 17(2): 378-386. https://doi.org/10.1021/ef020200r

[7] Su, M.M., Zhang, H.L., Zhang, Y.P., Zhang, Z.P. (2017). Miscibility and mechanical properties of SBS and asphalt blends based on molecular dynamics simulation. Journal of Chang'an University (Natural Science Edition), 37(3): 24-32. 8879.2017.03.004

[8] Zhang, L., Greenfield, M.L. (2007). Analyzing properties of model asphalts using molecular simulation. Energy \& Fuels,

21(3): 
https://doi.org/10.1021/ef060658j

[9] Zhang, L., Greenfield, M.L. (2008). Effects of polymer modification on properties and microstructure of model asphalt systems. Energy \& Fuels, 22(5): 3363-3375. https://doi.org/10.1021/ef700699p

[10] Li, D.D., Greenfield, M.L. (2011). High internal energies of proposed asphaltene structures. Energy \& Fuels, 25(8): 3698-3705. https://doi.org/10.1021/ef200507c

[11] Li, D.D., Greenfield, M.L. (2014). Chemical compositions of improved model asphalt systems for molecular simulations. Fuel, 115: 347-356. https://doi.org/10.1016/j.fuel.2013.07.012

[12] Wu, X.R. (2010). Synthesis and characterization analysis of poss hybrid nanocomposite. Harbin Institute of Technology.

[13] Wang, S.J., Zheng, Y.C., Ding, Y.J. (2013). Research on compatibility of monomer grafted SBS and asphalt based on molecular simulation technology. Highways \& Automotive Applications, 2013(1): 100-102. https://doi.org/10.3969/j.issn.1671-2668.2013.01.027

[14] Robertson, R.E., Branthaver, J.F., Harnsberger, P.M., Petersen, J.C., Dorrence, S.M., McKay, J.F., Turner, T.F., Pauli, A.T., Huang, S.C. (2001). Fundamental Properties of Asphalt and Modifified Asphalts, Volume I: Interpretive Report. See also FHWA-RD-99-213, Volume II: Final Report, New Methods.

[15] Painter, P.C. (1993). The characterization of asphalt and asphalt recyclability. Strategic Highway Research Program, National Research Council, 675: 1-5.

[16] Yan, R.F. (1991). New Progress in Research on Eucommia Gum Chemical Bulletin, 1991(1): 1-6. https://doi.org/10.14159/j.cnki.0441-3776.1991.01.001 